\title{
Trends and variability in stratospheric mixing: 1979-2005
}

\author{
H. Garny ${ }^{1}$, G. E. Bodeker ${ }^{2}$, and M. Dameris ${ }^{3}$ \\ ${ }^{1}$ Meteorological Institute, University of Munich, Munich, Germany \\ ${ }^{2}$ National Institute of Water and Atmospheric Research, Lauder, New Zealand \\ ${ }^{3}$ DLR-Institut für Physik der Atmosphäre, Oberpfaffenhofen, Germany
}

Received: 5 April 2007 - Published in Atmos. Chem. Phys. Discuss.: 10 May 2007

Revised: 9 August 2007 - Accepted: 30 October 2007 - Published: 14 November 2007

\begin{abstract}
Changes in climate are likely to drive changes in stratospheric mixing with associated implications for changes in transport of ozone from tropical source regions to higher latitudes, transport of water vapour and source gas degradation products from the tropical tropopause layer into the mid-latitude lower stratosphere, and changes in the meridional distribution of long-lived trace gases. To diagnose long-term changes in stratospheric mixing, global monthly fields of Lyapunov exponents were calculated on the $450 \mathrm{~K}, 550 \mathrm{~K}$, and $650 \mathrm{~K}$ isentropic surfaces by applying a trajectory model to wind fields from NCEP/NCAR reanalyses over the period 1979 to 2005 . Potential underlying geophysical drivers of trends and variability in these mixing fields were investigated by applying a least squares regression model, which included basis functions for a mean annual cycle, seasonally dependent linear trends, the quasibiennial oscillation (QBO), the solar cycle, and the El Niño Southern Oscillation (ENSO), to zonal mean time series of the Lyapunov exponents.

Long-term positive trends in mixing are apparent over southern middle to high latitudes at $450 \mathrm{~K}$ through most of the year, while negative trends over southern high latitudes are apparent at $650 \mathrm{~K}$ from May to August. Wintertime negative trends in mixing over northern mid-latitudes are apparent at $550 \mathrm{~K}$ and $650 \mathrm{~K}$. Over low latitudes, within $40^{\circ}$ of the equator, the QBO exerts a strong influence on mixing at all three analysis levels. This QBO influence is strongly modulated by the annual cycle and shows a phase shift across the subtropical mixing barrier. Solar cycle and ENSO influences on mixing are generally not significant. The diagnosed long-term changes in mixing should aid the interpretation of trends in stratospheric trace gases.
\end{abstract}

Correspondence to: H. Garny

(hella.garny@dlr.de)

\section{Introduction}

Stratospheric mixing, and in particular meridional barriers to stratospheric mixing, play a dominant role in the distribution of long-lived atmospheric trace gases on isentropic surfaces (Plumb and Ko, 1992). For example, changes in total column ozone in the Northern Hemisphere have been shown to be linked to interannual variability in lower stratospheric planetary wave breaking (Randel et al., 2002; Hood et al., 1999).

A number of different diagnostics of stratospheric mixing have been developed. The morphology of mixing on isentropic surfaces can be diagnosed from maps of potential vorticity (PV) since PV is a conservative quantity for adiabatic transport and therefore PV contours approximate material contours for time scales where diabatic effects can be neglected. The approach has been used to reveal barriers to meridional mixing as steep gradients in PV fields, and to reveal the presence of filaments of high latitude air in midlatitudes as tongues of high PV (McIntyre and Palmer, 1984; Hoskins et al., 1985). A technique based on advection of material contours has been used to diagnose fine-scale features in mixing and shows very good agreement with observed tracer distributions (Waugh, 1993; Waugh et al., 1994). A more recently developed quantity is the effective diffusivity, a modified Lagrangian mean diagnostic, which is derived by transforming the tracer transport advection-diffusion equation into a diffusion-only equation by introducing coordinates based on isolines of tracer mixing ratio (Nakamura, 1996; Allen and Nakamura, 2001; Haynes and Shuckburgh, 2000). Effective diffusivity is then a measure of the geometric complexity of tracer contours. The analysed tracer field can be based on observations (e.g. Nakamura and Ma, 1997) or on an artificial "test tracer" that is advected by analysed winds (e.g. Shuckburgh et al., 2001; Haynes and Shuckburgh, 2000). Another approach to measure mixing

Published by Copernicus Publications on behalf of the European Geosciences Union. 
is the use of finite-time Lyapunov exponents which measure the separation of two trajectories with time from initially nearby starting points. The exponents are related to the local stretching deformation of the fluid following an air parcel and have been used to identify mixing and barriers to mixing in the atmosphere (Pierrehumbert and Yang, 1993; Bowman, 1993). Finite-time Lyapunov exponents are derived from the more general concept of Lyapunov exponents which are defined as limit to infinite times and are originated in chaos theory, where they are used as a measure of sensitivity to perturbations in the initial conditions of a dynamical system, i.e. as a measure of chaos. The transition from infinite to finite times allows the use of the concept of Lyapunov exponents as a measure of local mixing rates. Next to finite-time Lyapunov exponents, other methods of generalizing the Lyapunov exponent concept to diagnosing mixing have been developed, for example finite-size Lyapunov exponents (Joseph and Legras, 2002) and deformation exponents (Koh and Plumb, 2000). In the study reported on here the method of finite-time Lyapunov exponents is used.

Using the diagnostics discussed above, stratospheric mixing has been investigated intensively and studies reveal inhomogeneities in mixing determined by regions of strong mixing and barriers to air exchange. Strong barriers to mixing have been identified at the polar vortex edge (McIntyre and Palmer, 1984; Bowman, 1993). The Antarctic vortex shows a more persistent barrier compared to its Arctic counterpart (Allen and Nakamura, 2001; Haynes and Shuckburgh, 2000). In the winter mid-latitudes, mixing is generally found to be strong. McIntyre and Palmer (1984), who coined the term "surf-zone" for this region, suggested that breaking of planetary-scale Rossby-waves, that can penetrate vertically from the troposphere into the stratosphere when winds are westerly (and therefore primarily in winter), are the cause of high winter-time mixing. Breaking of planetary waves is characterized by rapid and irreversible deformation of material contours. The contours form tongues that become longer and thinner with time, more and more fine-scaled, until the material is diffused into its surroundings (Tan et al., 1998). The "surf-zone" is bounded on its equatorward edge by a barrier to mixing with tropical air. In the absence of episodic mixing events (Waugh, 1993), this barrier separates tropical air from air at higher latitudes and creates a "tropical reservoir" for tracers that are transported into the stratosphere through the tropical tropopause (Trepte and Hitchmann, 1992). The summer hemisphere is generally characterized by weak mixing, since easterly winds prohibit vertical propagation of planetary waves. Changes in this spatial pattern of mixing have been studied previously. For example, Shuckburgh et al. (2001) investigated the influence of the quasi-biennial oscillation (QBO) on inter-annual changes in tropical mixing over a period of six years.

The goal of this paper is to quantify long-term (19792005) trends and variability in stratospheric mixing by applying a regression model to diagnostics of mixing. Potential explanatory factors of the variability, such as the QBO and solar cycle, are investigated by including indices for these factors as basis functions in the regression model. The calculation of the Lyapunov exponents used to diagnose mixing in this study is described in Sect. 2. The regression model used to quantify the trends and variability in the calculated global monthly Lyapunov exponents is described in Sect. 3. Examples of hemispheric distributions and zonal means of the calculated Lyapunov exponents are presented in Sect. 4 while the results from the application of the regression model are presented in Sect. 5. A discussion of these results, and the conclusions drawn, are presented in Sect. 6.

\section{Lyapunov exponents}

In an n-dimensional system, the spectrum of Lyapunov exponents is defined as:

$$
\lambda_{i}=\lim _{\substack{t \rightarrow \infty \\\left|\delta x_{i}\left(t_{0}\right)\right| \rightarrow 0}} \frac{1}{t-t_{0}} \ln \frac{\left|\delta x_{i}(t)\right|}{\left|\delta x_{i}\left(t_{0}\right)\right|} \quad(i=1 \ldots n)
$$

where the $\lambda_{i}$ are the $n$ Lyapunov exponents with initial orientation $i$ of the distance vector $\left|\boldsymbol{\delta} \boldsymbol{x}_{\boldsymbol{i}}\left(\boldsymbol{t}_{\mathbf{0}}\right)\right|$ between the starting points of two nearby trajectories. The $\left|\delta x_{i}(t)\right|$ are the distances between the two trajectories at time $t$ (Bowman, 1993). The $n$ initial distance vectors are the principal axes of a n-dimensional infinitesimal volume element, which is deformed in the flow into a n-dimensional ellipsoid (Wolf et al., 1985). The exponents of the Lyapunov spectrum can then be put in order with $\lambda_{1}>\lambda_{2}>\ldots>\lambda_{n}$, so that the largest Lyapunov exponent $\lambda_{1}$ is associated with the direction of the most rapid expansion (the axes grow with $\exp \left(\lambda_{1} t\right)$ ), while the direction of the nth exponent is the direction of maximal contraction. $\lambda_{1}$ then characterizes the divergence with time of trajectories with initially nearby starting points, and therefore gives a measure of chaos. For any direction of the initial distance vector between the starting points of the trajectories, the distance will grow exponentially with a rate given by the largest Lyapunov exponent, since growth in the direction of the first Lyapunov exponent will quickly dominate the spectrum (Rosenstein et al., 1993). Due to this domination of the direction of the largest Lyapuonv exponent, when calculating the whole spectrum, reorthonormalization has to be applied periodically to prevent all axes growing in the direction of the largest Lyapunov exponent.

The realization of the calculation of Lyapunov exponents for finite times leads to the finite-time Lyapunov exponent (FTLE) (Pierrehumbert and Yang, 1993). The FTLE gives a measure of local deformation rates, while the calculation of infinite time limit Lyapunov exponents would result in a uniform distribution in a system that is well mixed for long times (Joseph and Legras, 2002). In the study presented here, FTLEs were calculated on isentropic surfaces reducing the problem to a 2 -dimensional system. Since the 
flow on isentropic surfaces is to a good approximation incompressible, the conservation of the area spanned by the two distance vectors (initially a circle, deformed to an ellipse) is accounted for and therefore no additional information is given by the second FTLE (Pierrehumbert and Yang, 1993). The condition of small distances between the trajectories $\left(\left|\boldsymbol{\delta} \boldsymbol{x}_{\boldsymbol{i}}\left(\boldsymbol{t}_{\mathbf{0}}\right)\right| \rightarrow 0\right)$ is maintained by periodically renormalizing the distance $\left|\delta \boldsymbol{x}_{\boldsymbol{i}}(\boldsymbol{t})\right|$ which is practically done by periodically resetting the distance between the trajectories $\left|\delta \boldsymbol{x}_{i}(\boldsymbol{t})\right|$ to $\left|\boldsymbol{\delta} \boldsymbol{x}_{\boldsymbol{i}}\left(\boldsymbol{t}_{\mathbf{0}}\right)\right|$ (Wolf et al., 1985). The FTLE with renormalization after a period $T$ is then given by:

$$
\lambda=\frac{1}{n T} \sum_{k=1}^{n} \ln \frac{\delta x(k T)}{\delta x(0)}
$$

where $\delta x(k T)$ is the distance after $k$ time intervals of length $T, n$ the total number of intervals and $\delta x(0)$ the initial distance at $t_{0}=0$. Figure 1 shows two example 12 day trajectories on the $450 \mathrm{~K}$ level, commencing on 1 August 2000, and where renormalization is applied after 6 days. Distances labelled A and B denote $\delta x(k T)$ (for $\mathrm{k}=1,2$ ).

For the purposes of this study, two dimensional (latitude and longitude) Lagrangian forward trajectories were computed on isentropic surfaces using a standard 4th order Runge-Kutta integration scheme (Press et al., 1989) applied at $1 \mathrm{~h}$ integration intervals to 6 hourly $2.5^{\circ} \times 2.5^{\circ}$ NCEP/NCAR wind fields on the 450,550 and $650 \mathrm{~K}$ isentropes. The zonal and meridional wind components were interpolated to the location of the advected air parcels using bilinear spatial interpolation and linear interpolation in time. Trajectories within $20^{\circ}$ of the poles were transformed to a Cartesian coordinate system to avoid the singularity at the pole which occurs when prescribing winds using zonal and meridional components.

McKenna et al. (2002), who used the FTLE as a tool to model mixing in a chemical lagrangian model, show that FTLEs are directly related to shear and strain rates of the flow. The relation between Lyapunov exponents and mixing, that can be parameterised as diffusivity, can be established in the following way (as shown by McKenna et al., 2002): since in the calculation of FTLE only differential and not mean advection is accounted for, the corresponding equation for tracer concentrations is a diffusion-only equation (by parametrizing the differential advection as a diffusion term), with a solution of the form $\exp \left(-\frac{x^{2}}{4 D t}\right)$ where $D$ is the diffusivity. By assuming that the distance $\delta x(T)$ represents the halfwidth of the tracer distribution after time step $T$, the diffusivity can be approximated by $\frac{\delta x(T)^{2}}{4 T}$. By replacing $\delta x(T)$ with $\delta x(0) \exp \lambda T$ the diffusivity can then be related to the FTLE $(D=$ const $\times \exp (\lambda))$, and a link between the physical process of mixing and Lyapunov exponents is established (even though this connection is rather conceptual). Effective diffusivity, a different measure to the diffusivity described above, has been used by Haynes and Shuckburgh (2000) to diagnose both stratospheric transport

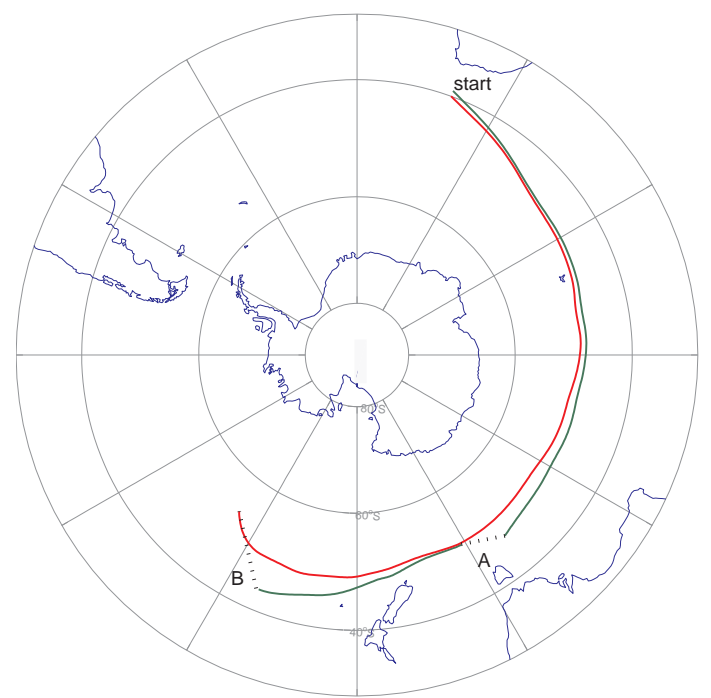

Fig. 1. Trajectories (red and green solid line) for a 12 day period on the $450 \mathrm{~K}$ level, commencing on 1 August 2000, and with renormalization after 6 days (A and B). The distances A and B, shown as dashed lines, are used to calculate Lyapunov exponents. To show more clearly the evolving spatial separation of the trajectories, the distance between the starting points has been set at $100 \mathrm{~km}$ compared to the $1 \mathrm{~km}$ value used in the calculations presented in this paper.

and mixing. While effective diffusivity broadly matches the spatial structure obtained with FTLE (which measures only mixing), disagreements in the detailed structure exist (Shuckburgh and Haynes, 2003). Shuckburgh and Haynes (2003) also stated that in cases where the air parcel is advected out of the region of interest within the time period used for the calculation of the FTLE, the resulting FTLE disagree with effective diffusivity fields since the FTLE no longer describe mixing in this specific region as effective diffusivity does, but the amount of mixing that an air parcel originating in this region will experience over the cause of the time period.

In this study, Lyapunov exponents, with units of day ${ }^{-1}$, were calculated from 30 day trajectories with a renormalization period of 10 days. Over this period, diabatic ascent and descent rates were assumed to be negligible (Bowman, 1993). The choice of the renormalization period is not critical (Wolf et al., 1985), and calculations of the Lyapunov exponents for varying $T$ were found to give similar results. The initial distance $\delta x(0)$ was chosen to be $1 \mathrm{~km}$, resulting in a separation of approximately $400 \mathrm{~km}$ after 30 days (taking $\lambda=0.2$ day $^{-1}$, as suggested by the results in Sect. 4 ), which is a sufficiently small scale compared to the scale of interest (e.g. the polar vortex). The trajectory length of 30 days provides Lyapunov exponents for each month. However, these are not monthly means but rather should be interpreted as the degree to which an air parcel is deformed, and therefore mixed with surrounding air, over the course of the month. 
The location identified with a particular Lyapunov exponent is the origin of the calculated trajectories. The resultant monthly Lyapunov exponents are presented in Sect. 4.

To investigate the effects of uncertainties in the NCEP/NCAR reanalyses wind fields on the calculated Lyapunov exponents, the calculations were repeated for one year (1984) with wind fields perturbed as follows: Gaussian distributed errors were added to the winds at each grid point, choosing a standard deviation for the $u$ component of $2.5 \mathrm{~m} \mathrm{~s}^{-1}$ and for the $\mathrm{v}$ wind component of $1.5 \mathrm{~m} \mathrm{~s}^{-1}$ based on the uncertainties reported in Kalnay et al. (1996). The resulting relative errors in the Lyapunov exponents are approximately normally distributed with a mean value near zero $(-0.0283)$ and a standard deviation of $\sim 0.12$. These results indicate a sufficient robustness against errors in the wind fields. The time-latitude pattern of zonal mean Lyapunov exponents of the runs with and without errors are well correlated (with a correlation coefficient of $\sim 0.72$ ).

\section{The regression model}

To quantify the underlying geophysical drivers of trends and variability in the calculated Lyapunov exponents, zonal means were calculated and analysed using a least squares regression model of the form

$$
\begin{aligned}
\lambda_{m}(\phi, t)= & a_{1}(\phi, t)+ \\
& a_{2}(\phi, t) \cdot \operatorname{Linear}(t)+ \\
& a_{3}(\phi, t) \cdot \mathrm{QBO}(t+\Delta t)+ \\
& a_{4}(\phi, t) \cdot \operatorname{Solar}(t)+ \\
& a_{5}(\phi, t) \cdot \operatorname{ENSO}(t)
\end{aligned}
$$

where $\phi$ is the latitude, $t$ is the time in months ( $t=1$ for January 1979), and $\lambda_{m}(\phi, t)$ is the modelled Lyapunov exponent. The regression model residuals $(r(\phi, t))$ are the differences between the modelled and calculated Lyapunov exponents. In addition to an offset and mean annual cycle, a linear term, the QBO, the solar cycle, and El Niño southern oscillation (ENSO) have been included as basis functions. Their coefficients $a_{i}$ were expanded to account for seasonal variability as follows:

$$
\begin{array}{r}
a_{i}(\phi, t)=a_{i 0}(\phi)+\sum_{k=1}^{N}\left[a_{\mathrm{iks}}(\phi) \sin (2 \pi k(t-0.5) / 12)+\right. \\
\left.+a_{\mathrm{ikc}}(\phi) \cos (2 \pi k(t-0.5) / 12)\right]
\end{array}
$$

$N$ is set to 4 for the constant term $a_{1}$, and for all other basis functions it is set to 2 , so that the total number of coefficients is 29 . Setting $N$ to 4 for the constant term allows for accurate representation of the annual cycle which is simply the Fourier expansion of the constant term, while restricting $N$ to 2 for the other terms allows for annual and semi-annual structure in the coefficients without over-fitting the regression model. The coefficients were calculated by minimizing the sum of the squared residuals for each latitude separately. Uncertainties in the regression coefficients were calculated using:

$$
\sigma_{a_{k}}^{2}=\frac{\chi^{2}}{N-M}\left[\left(\mathbf{X}^{T} \mathbf{X}\right)^{-1}\right]_{k k}
$$

where $\chi^{2}$ is the sum of the squared residuals, $N$ is the length of the time series, $M$ is the number of coefficients, and $\mathbf{X}$ is the matrix of basis functions (von Storch and Zwiers, 2002). This method of calculating the uncertainties in the regression coefficients assumes that the residuals are white noise i.e. normally distributed with zero mean and statistically independent in time. If this assumption is violated, the uncertainties on the model coefficients will be significantly underestimated (Tiao et al., 1990). In these circumstances a more accurate estimate of the uncertainties in the parameters must be calculated by correcting the residual term for its autocorrelation. The method described in Appendix A of Tiao et al. (1990) is applied here. The first step is to run the regression model and obtain the coefficients $a_{1}$ to $a_{5}$. These coefficients, together with the basis functions, are then used to construct the modelled Lyapunov exponents which are then subtracted from the observed values to derive the residuals. A first order autocorrelation coefficient $(\varrho)$ is derived by regressing the residuals at time step $t$ against the residuals at time $t-1$. This coefficient is then used to transform the time series $\lambda(\phi, t)$, and the basis functions, as follows:

$$
x(t) \rightarrow x(t)-\varrho \cdot x(t-1)
$$

The regression model is now rerun with the transformed variables. The corrected variance in the coefficients is obtained as described above (see Eq. 4) with the transformed model. The uncertainty estimates when taking autocorrelation into account were almost twice as high as when neglecting it, which shows the necessity for doing so in this case. The total variance for coefficient $a_{i}$ is obtained by

$$
\begin{aligned}
\sigma^{2}\left(a_{i}\right)= & \sigma^{2}\left(a_{i 0}\right)+\sum_{k=1}^{N}\left[\sigma^{2}\left(a_{\mathrm{iks}}\right) \sin ^{2}(2 \pi k(t-0.5) / 12)\right]+ \\
& \left.+\sigma^{2}\left(a_{\mathrm{ikc}}\right) \cos ^{2}(2 \pi k(t-0.5) / 12)\right]
\end{aligned}
$$

In the following, coefficients are considered as significant when $a_{i} \geq 2 \sigma\left(a_{i}\right)$, which corresponds to a significance level of about $95 \%$ (the ratio $a_{i} / \sigma\left(a_{i}\right)$ is student-t distributed and converges to the normal distribution for $N-M \geq 30$ ). This means that with a certainty of $95 \%$ the null hypothesis $a_{i}=0$ can be rejected if $a_{i} / \sigma\left(a_{i}\right)>2$.

The portion of variability in Lyapunov exponents that is explained by the fitted model can be estimated by the coefficient of multiple determination $R^{2}$ (von Storch and Zwiers, 2002), which is given by

$$
R^{2}(\phi)=\frac{\sum_{i=1}^{N}\left(\lambda_{m}(\phi, i)-\overline{\lambda(\phi)}\right)^{2}}{\chi^{2}+\sum_{i=1}^{N}\left(\lambda_{m}(\phi, i)-\overline{\lambda(\phi)}\right)^{2}}
$$




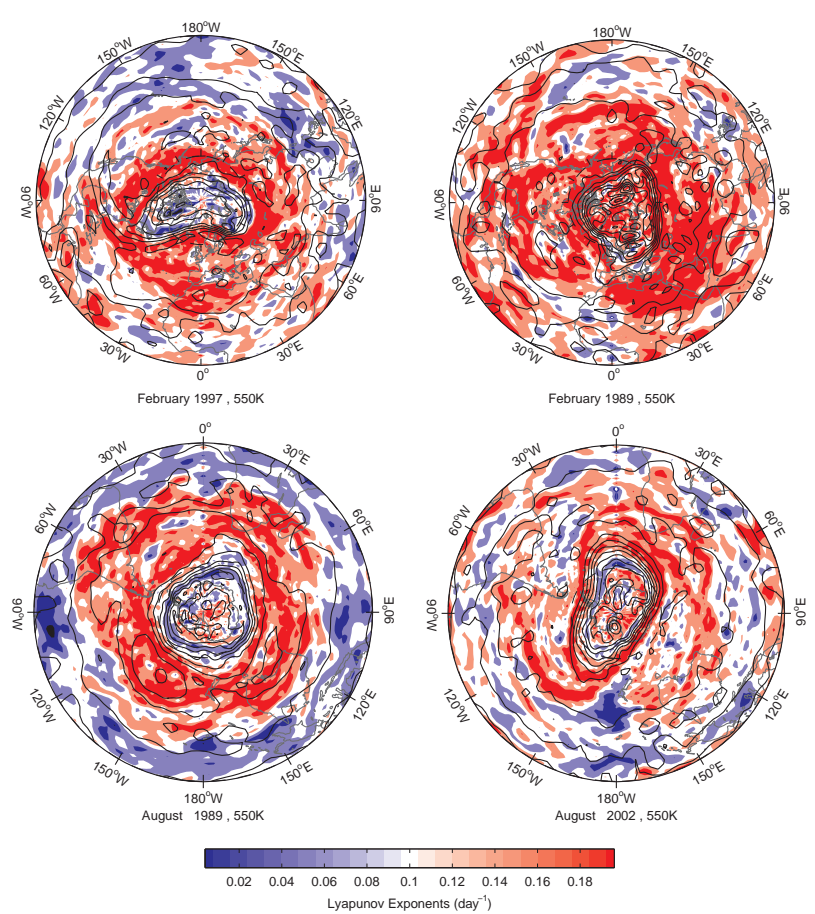

Fig. 2. Example Lyapunov exponents for February in the Northern Hemisphere (upper panels) and for August in the Southern Hemisphere (lower panels), on the $550 \mathrm{~K}$ surface. For each hemisphere, results from two years are presented and compared. Regions coloured red show high mixing while regions coloured blue show low mixing. Overlaid on the Lyapunov distributions are the $550 \mathrm{~K}$ PV fields, shown as contour plots, at the start time of the trajectories used to calculate the Lyapunov exponents.

where $\overline{\lambda(\phi)}$ is the mean observed Lyapunov exponent at latitude $\phi$.

The basis functions used for this regression model are indicators of processes that are believed to play a role in the variability in mixing. The first term on the right hand side of Eq. (2) simply represents the mean annual cycle and offset. By choosing $N=4$ in Eq. (3) periodicities of 12, 6, 4 and 3 months are allowed. The second term represents a linear trend in mixing, and with $N=2$, allows for trends to be different during different months of the year with annual and semi-annual structure.

The QBO dominates equatorial dynamics and is highly likely to influence mixing (Shuckburgh et al., 2001). Monthly mean equatorial zonal mean winds at $50 \mathrm{hPa}$ from the NCEP/NCAR reanalyses were used as the QBO basis function, where a time lag $\Delta t$ was allowed. The optimal value for $\Delta t$ was identified by taking the best fit (minimal sum of squared residuals) of runs with $\Delta t$ varying from -12 to +12 months.

The 11-year solar cycle shows significant correlations with dynamical quantities such as geopotential height and temperature in the lower stratosphere (van Loon and Labitzke, 2000). A solar cycle influence on mixing is therefore con-
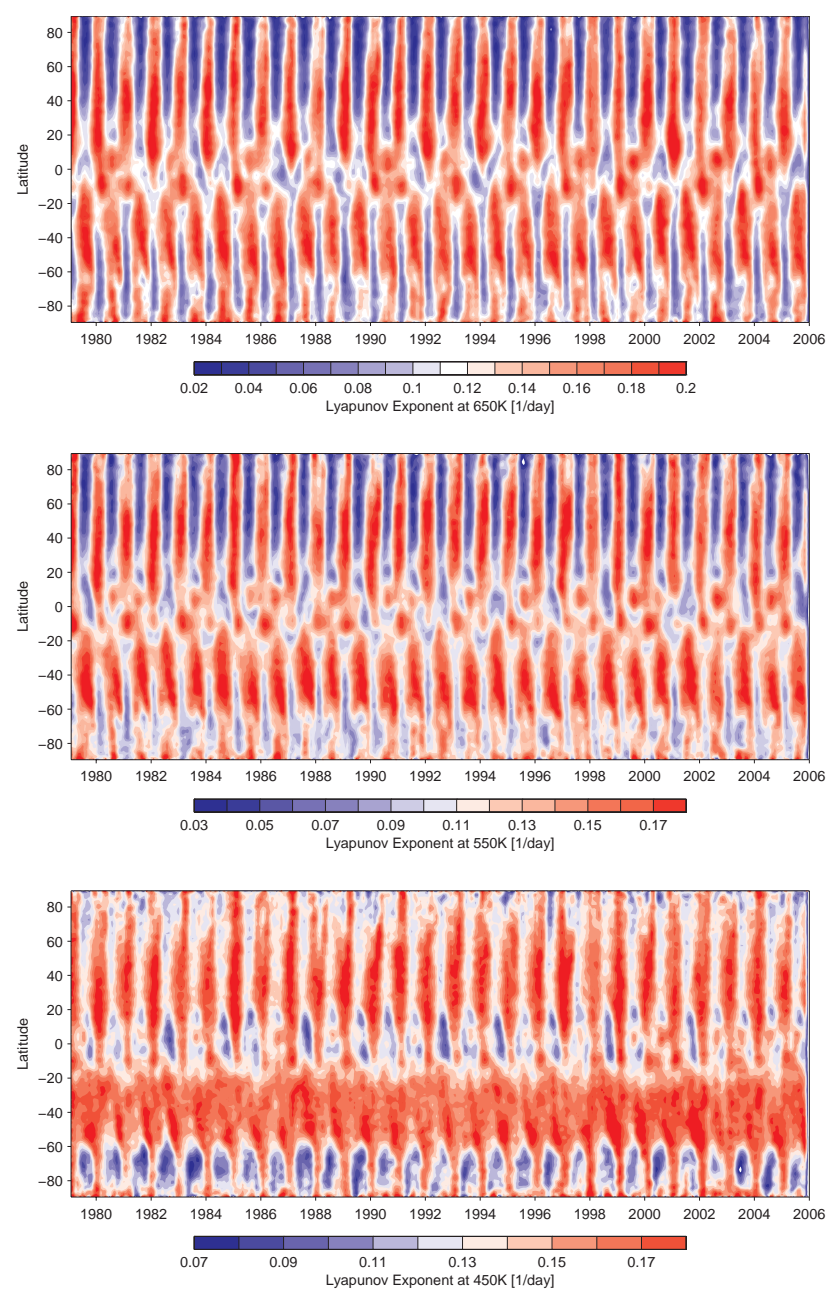

Fig. 3. Zonally averaged Lyapunov exponents for $650 \mathrm{~K}$ (top), $550 \mathrm{~K}$ (middle) and $450 \mathrm{~K}$ (bottom) for years 1979-2005. Note the different colour scales.

ceivable and has been incorporated in the regression model as $10.7 \mathrm{~cm}$ solar fluxes measured at Ottawa.

ENSO is represented by the southern oscillation index (SOI), the difference in monthly mean sea level pressure between Tahiti and Darwin.

All basis functions were normalized to the range $[-1 ; 1]$ by linear transformation.

\section{Results from Lyapunov exponent calculations}

Lyapunov exponents were calculated on a latitude-longitude grid of $180 \times 180$ points to provide global coverage at $1^{\circ}$ latitude and $2^{\circ}$ longitude resolution. Examples of hemispheric distributions of the calculated Lyapunov exponents are shown in Fig. 2.

Because the Lyapunov exponents shown in Fig. 2 integrate the effects of mixing over a one month period, features with 


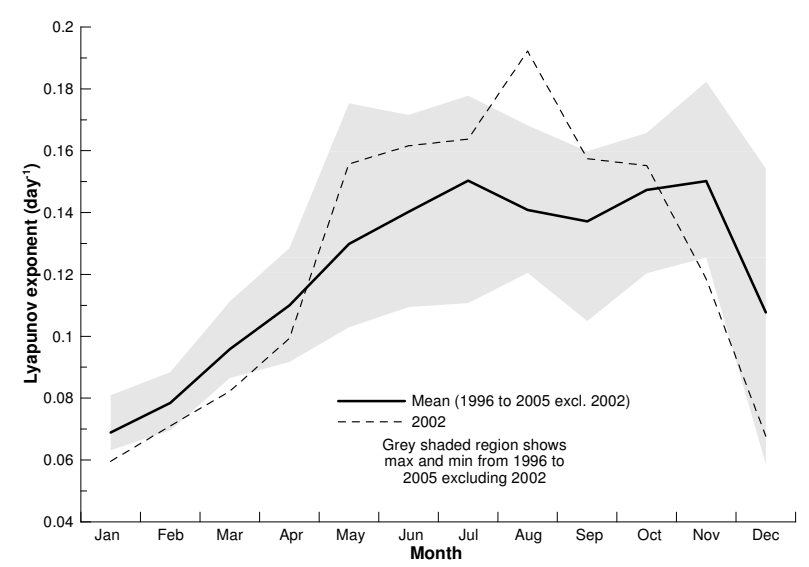

Fig. 4. Lyapunov exponents averaged over $60^{\circ} \mathrm{S}$ to $90^{\circ} \mathrm{S}$ at $650 \mathrm{~K}$ for 2002 (dashed line). The 10-year mean 1996 to 2005 (excluding 2002) is shown as a solid line together with the range of values over this time period (grey shaded region).

a shorter time-scale, such as tongues of PV in mid-latitudes apparent in the instantaneous PV fields at the start date of the trajectories, would not be reflected in the Lyapunov exponent distributions. However, where features in the PV field persist over time scales on the order of 1 month, the extent of mixing associated with those features is reflected in the Lyapunov exponent distributions.

In 1996/1997 the Arctic vortex was weak early in the season and grew rapidly in strength from early 1997 onwards (see further discussion below). In contrast, in 1988/1989 the vortex was strong early in the winter season but was significantly disturbed by a sudden stratospheric warming in February 1989. While steep gradients are still visible in the PV field at the start of February, the Lyapunov exponents show strong mixing of air from the interior of the vortex to midlatitudes. In 1989, the Antarctic vortex was strong and well formed by August. In 2002 the early August vortex was very elongated and the Lyapunov exponents suggest that there was mixing across the vortex edge (the region of steep gradients in PV).

For the analyses presented below, the Lyapunov exponents were zonally averaged, based on their starting coordinates, and are shown in Fig. 3 for 1979 to 2005. Note the different colour scaling in the three panels, resulting from increasing variability in mixing with height. A clear annual cycle can be seen at all levels, and in the mid-latitudes mixing is generally high in winter and low in summer. Over southern midlatitudes at $450 \mathrm{~K}$, the annual cycle is very weak. At $550 \mathrm{~K}$ and $650 \mathrm{~K}$, the region of high mixing in winter mid-latitudes, the stratospheric "surf zone", is equally strong in both hemispheres but extends over a greater range of latitudes in the Northern Hemisphere. At these two levels, mixing is weaker in summer in the Northern Hemisphere than in the Southern Hemisphere.

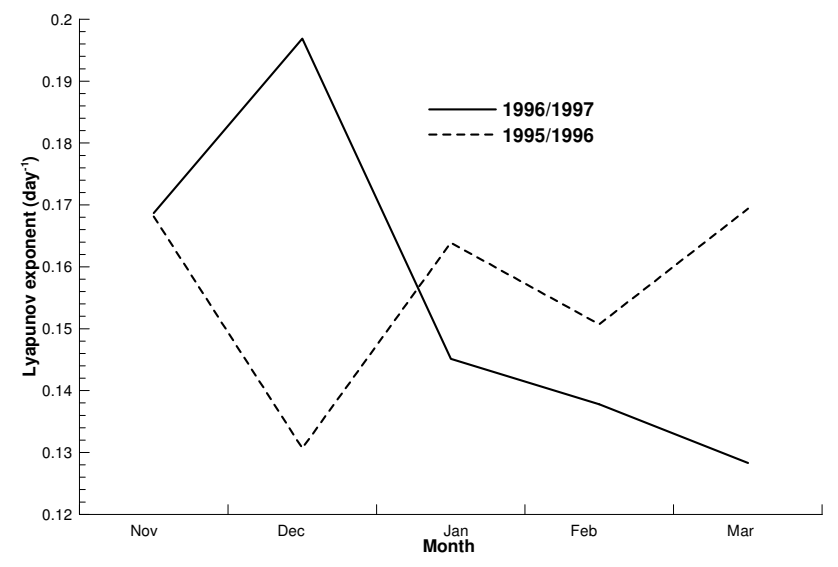

Fig. 5. Lyapunov exponents averaged over $60^{\circ} \mathrm{N}$ to $90^{\circ} \mathrm{N}$ at $450 \mathrm{~K}$ for the northern winters 1995/96 (dashed line) and 1996/97 (solid line).

The Antarctic polar vortex is clearly visible as a wintertime region of low mixing poleward of $\sim 60^{\circ} \mathrm{S}$ at the $450 \mathrm{~K}$ level. At $550 \mathrm{~K}$ and $650 \mathrm{~K}$ the Antarctic polar barrier shows low mixing close to the vortex edge with higher mixing both poleward and equatorward of the edge, with considerable interannual variability. The disturbed Antarctic vortex of 2002 (Newman and Nash, 2005) appears as a patch of reduced weak mixing during the 2002 winter at $450 \mathrm{~K}$. At $550 \mathrm{~K}$ and $650 \mathrm{~K}$ mixing in late autumn and winter is stronger than usual, and shows an anomalously early transition to even stronger mixing (as shown for $650 \mathrm{~K}$ in Fig. 4).

In the Northern Hemisphere the polar vortex is apparent only in some years, e.g. 1989/1990, 1996/1997 and 1999/2000 while in other years, e.g. 1984/1985 and $1998 / 1999$, the winter-time mixing extends all the way to the pole. In the Arctic winter of 1996/1997 the vortex did not form until late December after which it was strong and centred on the pole until a late breakdown in April (Coy et al., 1997). This behaviour is apparent at $450 \mathrm{~K}$ in Fig. 5 where a sharp transition from strong mixing in December to weak mixing in January in the Arctic can be seen. This transition is also apparent, but less clearly, at $550 \mathrm{~K}$ and $650 \mathrm{~K}$ (not shown). The opposing seasonal patterns of change in the Arctic winters of 1995/1996 (weak to strong mixing) and 1996/1997 (strong to weak mixing) are reflected in minimum Arctic temperatures on the $475 \mathrm{~K}$ surface and in the strength the jet (Figs. 1 and 2 of Coy et al., 1997).

In the tropics, the annual cycle is still apparent but superimposed by interannual differences, especially at upper levels. At $450 \mathrm{~K}$ the "tropical reservoir" (Trepte and Hitchmann, 1992), a region of low mixing within $\sim 15^{\circ}$ of the equator, is apparent each year with varying strength while at the upper levels, regions of high and low mixing show a more complex structure. At the two upper levels, some two-year periodicity can be seen in the tropical mixing, indicating influence of the QBO. 

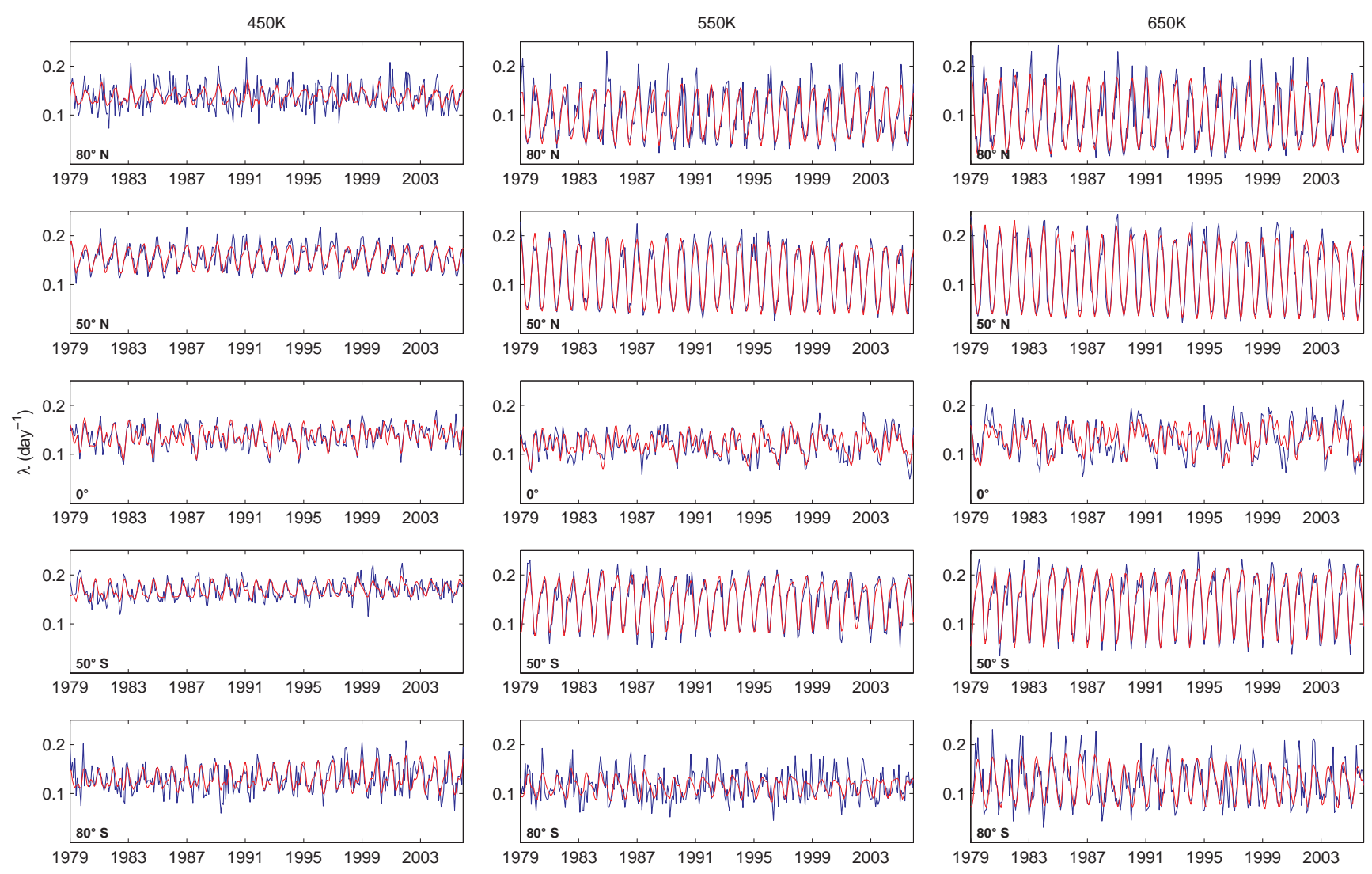

Fig. 6. Time series of calculated (blue) and modelled (red) Lyapunov exponents for latitudes $80^{\circ} \mathrm{N} / \mathrm{S}, 50^{\circ} \mathrm{N} / \mathrm{S}$, and the equator. Left panels show regression model fits for $450 \mathrm{~K}$, middle panels for $550 \mathrm{~K}$ and right panels for $650 \mathrm{~K}$.

\section{Regression model analysis}

\subsection{Regression model fits}

The regression model described in Sect. 3 was applied to the zonal mean monthly Lyapunov exponent time series at each degree of latitude from 1979 to 2005. Examples of regression model fits at 5 selected latitudes and all three analysis levels are shown in Fig. 6. At latitudes of $50^{\circ}$ north and south the variability is dominated by the annual cycle and the model captures the variability well (coefficients of multiple determination $R^{2}$ as given by Eq. 5 are $0.93,0.88,0.93$ and 0.92 ) at the two upper levels. At $450 \mathrm{~K}$ in the mid-latitudes the annual cycle is less pronounced, and less variability is explained by the model ( $R^{2}$ values of 0.69 and 0.51$)$. In the polar regions $\left(80^{\circ} \mathrm{N} / \mathrm{S}\right)$, at all three levels, the year-to-year variability is much higher and the regression model has difficulty tracking the observations. At $550 \mathrm{~K}$ in the Northern Hemisphere, and at $650 \mathrm{~K}$ in both hemispheres, the observed Lyapunov exponents show a strong annual cycle and the regression model captures much of the variance - note however, that the large Arctic winter-time mixing in 1985, 1989, and 2001 is not fully captured by the regression model, suggesting that there are additional sources of variability not currently accounted for. At $550 \mathrm{~K}$ in the Southern Hemisphere, and at $450 \mathrm{~K}$ in both hemispheres, the annual cycle is very weak and the regression model has difficulty tracking the observations $\left(R^{2}\right.$ values of $0.29,0.48$ and 0.36 ). At the equator, the model fits the variability in the observed Lyapunov exponent time series very well $\left(R^{2}\right.$ values close to 0.71$)$.

\subsection{Lyapunov exponent climatology}

The $a_{i}$ coefficients in Eq. (2) have been expanded at daily resolution (see Eq. 3) at each latitude and coefficients 1 to 3 are plotted in Figs. 7, 8 and 9 for the three isentropic levels. The first coefficient ( $a_{1}$, uppermost panel) represents the climatology in Lyapunov exponents for years 1979-2005 and was significant $\left(a_{i}>2 \sigma_{a_{i}}\right.$, see Sect. 3) for all latitudes and times.

The climatology for $450 \mathrm{~K}$ shows high winter-time values of Lyapunov exponents in the mid-latitudes of both hemispheres. In the mid-latitudes of the Southern Hemisphere the annual cycle in mixing is small and values remain high year round with a maximum in spring (September to November) at around $60^{\circ} \mathrm{S}$ in approximate agreement with Allen and Nakamura (2001) and Haynes and Shuckburgh (2000). Throughout the Northern Hemisphere, however, we find 

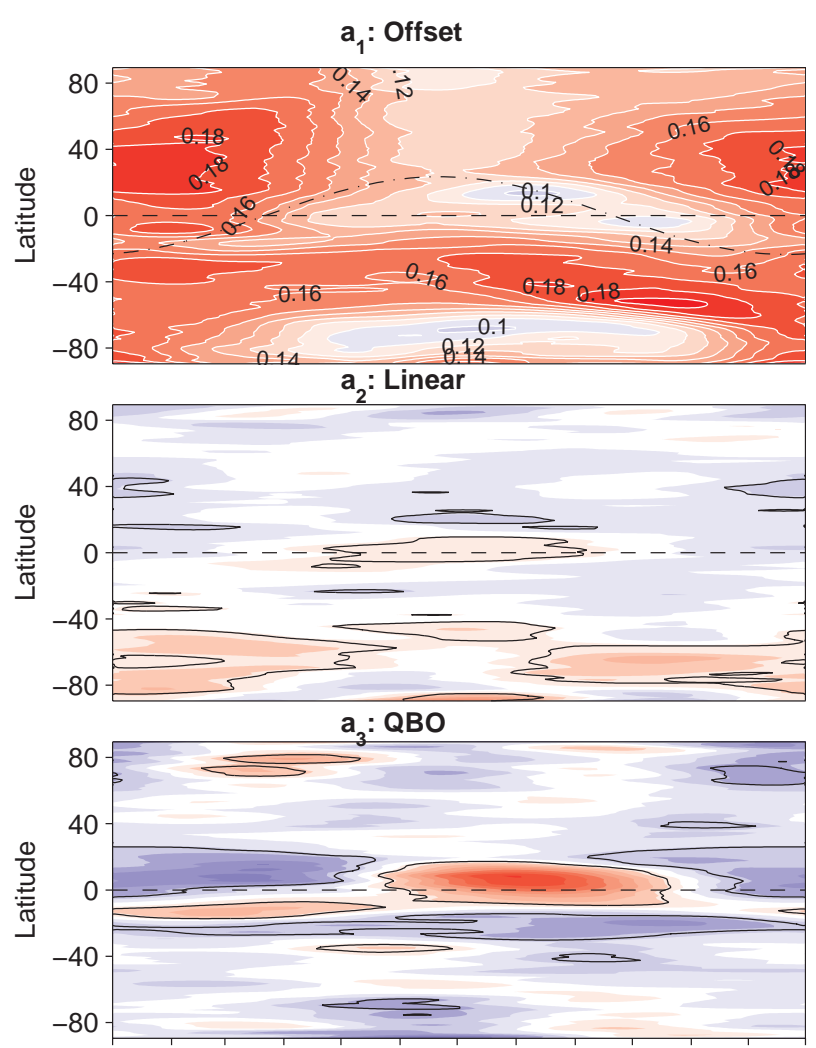

Jan Feb Mar Apr May Jun Jul Aug Sep Oct Nov Dec

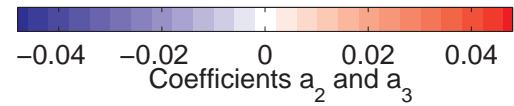

Fig. 7. Regression model coefficients at $450 \mathrm{~K}$ as given by equation 3 but evaluated daily. The uppermost panel shows the climatology of mixing $\left(a_{1}\right)$, with values indicated by the contour labels. The coefficient $a_{1}$ is significant ( $a_{1}>2 \sigma_{a_{1}}$, see text) for all latitudes and times. The dash-dotted line denotes the solar declination. For the two lower panels, showing the coefficients of the linear term $\left(a_{2}\right)$ and the QBO term $\left(a_{3}\right)$, the colour bar at the bottom shows the scaling. Parameters are significant within the black contours.

weaker mixing in summer than in winter which disagrees with Allen and Nakamura (2001) and Haynes and Shuckburgh (2000) who found strong mixing at $450 \mathrm{~K}$ in northern summer.

The tropics are characterized by a broad band of low values from May to November, which is shifted slightly toward the Northern Hemisphere. From December to April there is a narrow band of low mixing at about $15^{\circ} \mathrm{S}$. This shift toward the summer pole is also apparent in the 18 month time series of Haynes and Shuckburgh (2000), even though they find a broad band of low mixing year round.

The Antarctic vortex appears as a region of very low Lyapunov exponents with steep gradients across $60^{\circ} \mathrm{S}$. Mixing is low within the vortex, in agreement with Haynes and Shuckburgh (2000) and Bowman (1993). This strong barrier develops in late autumn (April-June) and remains strong un-
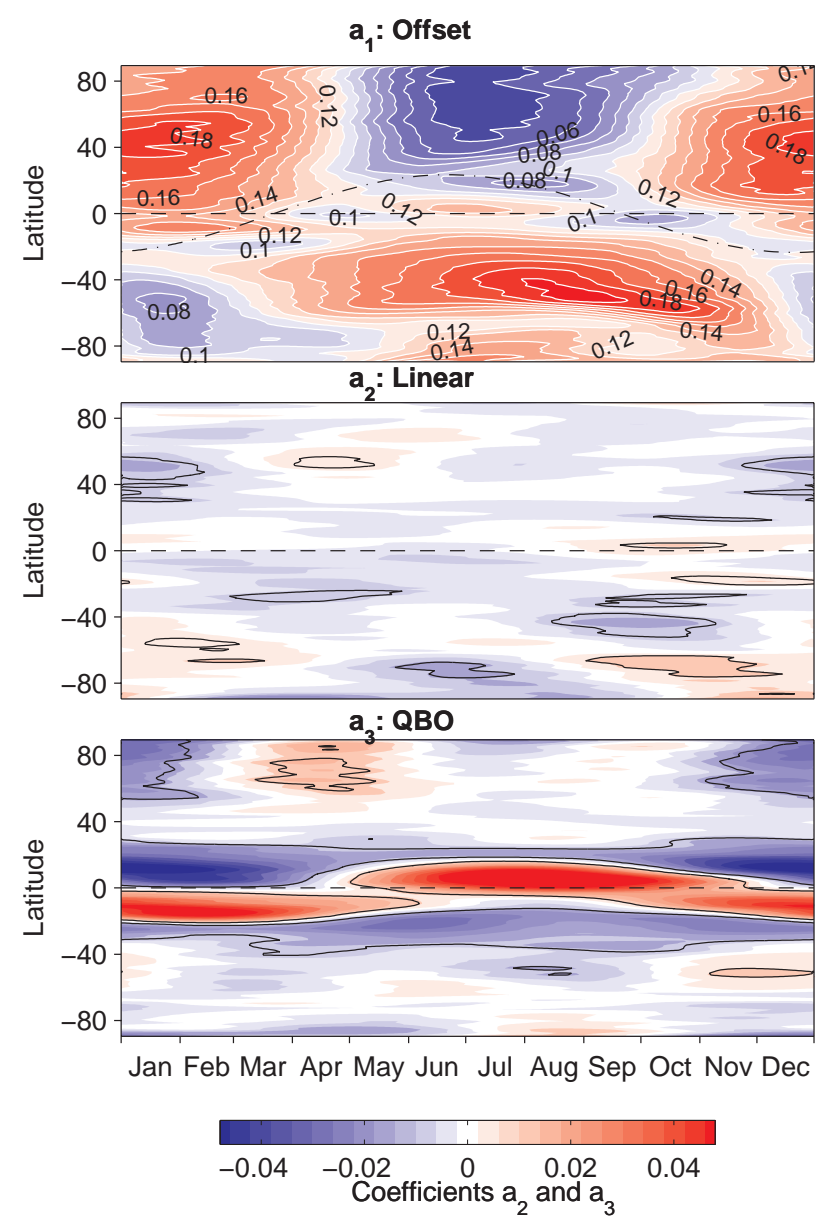

Fig. 8. As in Fig. 7 but for $550 \mathrm{~K}$.

til the vortex breaks up in spring (October-December). The maximum in mixing is located at the vortex edge in October/November, which coincides with erosion of the polar vortex by breaking planetary waves. In the Arctic mixing is lowest in summer, and the Arctic vortex is hardly apparent in zonally averaged Lyapunov exponents.

The climatologies at $550 \mathrm{~K}$ and $650 \mathrm{~K}$ show many similarities. The high mixing in the surf-zone in winter and low mixing in summer mid-latitudes appear at both levels, with a stronger annual cycle at $650 \mathrm{~K}$. Also at both levels the summer minimum extends to the pole in the northern but not in the Southern Hemisphere. The tropical climatology at these two upper levels shows a seasonally consistent spatial pattern whereby regions of generally low mixing follow a path that is close to, but not perfectly coincident with, the solar declination (dash-dot line in Fig. 8). However, the mixing is not uniform in time along this path and 5 periods of low mixing are interspersed with periods of higher mixing. In the summer hemisphere, a region of higher mixing occurs equatorward of the regions of low mixing, but appears confined to the tropics and separated from the main winter-time region of high 

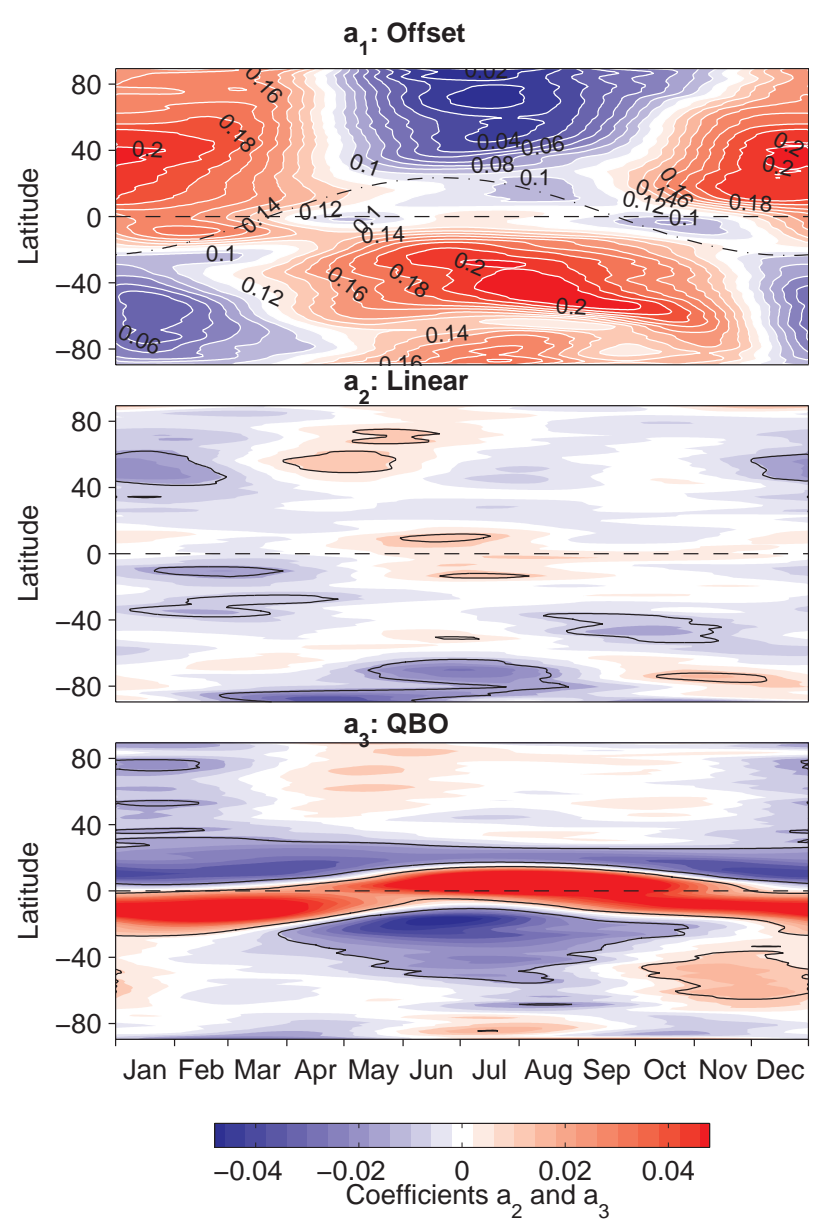

Fig. 9. As in Fig. 7 but for $650 \mathrm{~K}$.

mixing in the mid-latitudes. The pattern at $650 \mathrm{~K}$ is similar to the pattern at $550 \mathrm{~K}$ but with longer lasting maxima and suppressed minima. Due to the large influence of the QBO in the tropics (see Sect. 5.4), these tropical mixing patterns are unlikely to be directly comparable to other studies which consider only a few years of data and show time series rather than climatologies (e.g. Haynes and Shuckburgh, 2000).

In contrast to $450 \mathrm{~K}$, there are high values of Lyapunov exponents within the fully developed Antarctic vortex in midwinter. The polar barrier is visible as a band of lower values south of $60^{\circ} \mathrm{S}$. The barrier appears stronger at $550 \mathrm{~K}$ than at $650 \mathrm{~K}$ and low mixing extends all the way to the pole just before the final warming mixes air from within the vortex into mid-latitudes in early summer. This behaviour is again in good agreement with Haynes and Shuckburgh (2000). As at $450 \mathrm{~K}$, the Arctic vortex is seen only weakly.

\subsection{Trends in mixing}

The $a_{2}$ coefficients from Eq. (2) have been used to describe global, seasonally dependent, long-term (1979-2005) trends in mixing at $450 \mathrm{~K}, 550 \mathrm{~K}$ and $650 \mathrm{~K}$. At $450 \mathrm{~K}$, the domi-

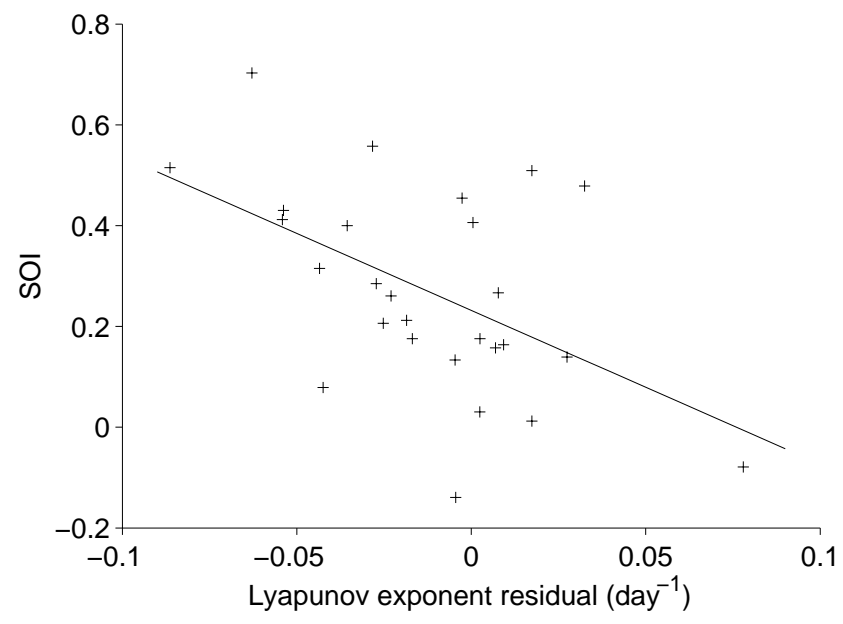

Fig. 10. Lyapunov exponents at $650 \mathrm{~K}$ with all fitted basis function other than ENSO removed (i.e. subtracting terms 1 to 4 in Eq. 2 from the original time series) and averaged over April-June and over $30^{\circ}$ to $60^{\circ} \mathrm{N}$ plotted against the SOI for each year from 1979 to 2005 . The solid line is the linear fit to the data points.

nant pattern is a positive trend in mixing in middle and high southern latitudes with highest values in summer. The maximum total change in mixing of $0.03 \mathrm{day}^{-1}$ over the whole period of 27 years is found at $\sim 65^{\circ} \mathrm{S}$ from mid-September to mid-November, suggesting that mixing across the vortex edge on the $450 \mathrm{~K}$ surface has increased over this period. Between $40^{\circ} \mathrm{S}$ and $60^{\circ} \mathrm{S}$ there are also indications of increases in mixing from January to July. In a confined latitude band close to the equator, positive trends in mixing are seen from May to August.

At $650 \mathrm{~K}$ both positive and negative trends in mixing are apparent, and the main features occur also at $550 \mathrm{~K}$ in addition to some barely significant trends with little latitudinal extent. In the northern surf zone, a total winter-time change of $-0.03 \mathrm{day}^{-1}$ occurs at $650 \mathrm{~K}$ over the 1979-2005 period. At $650 \mathrm{~K}$ (and less so at $550 \mathrm{~K}$ ), increases in mixing over northern mid-latitudes occur in late spring and early summer. In contrast spring-time decreases in southern midlatitudes are seen. Over southern high latitudes, mixing increases in spring/summer and decreases in winter within the vortex. The decreases in winter-time Antarctic mixing are more apparent at $650 \mathrm{~K}$ than at $550 \mathrm{~K}$ with a total long-term change of $-0.05 \mathrm{day}^{-1}$. In contrast to $450 \mathrm{~K}$, this implies a strengthening of the polar barrier but at an earlier time in the year (July). Over southern high latitudes, increases in mixing are seen at around the time when the vortex breaks up, more so at $550 \mathrm{~K}$ than at $650 \mathrm{~K}$.

\subsection{QBO influence on mixing}

The QBO is found to account for most of the non-seasonal variability in Lyapunov exponents in the tropics, as the third panel in Figs. 7 to 9 show. The response of mixing to the 


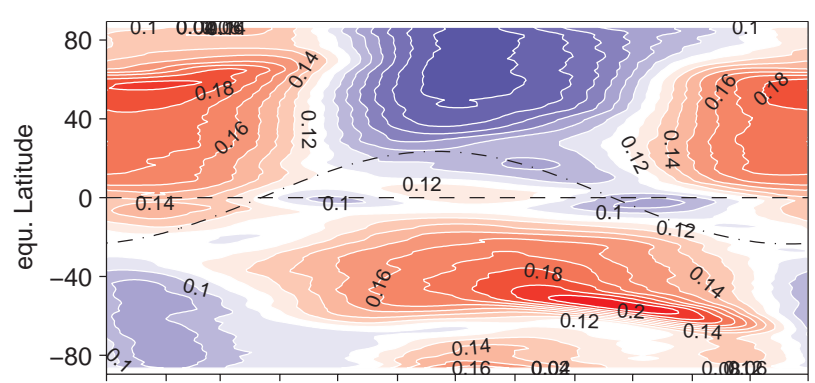

Jan Feb Mar Apr May Jun Jul Aug Sep Oct Nov Dec

Fig. 11. Climatology of Lyapunov exponents (i.e. regression model coefficient $a_{1}$ ) averaged over equivalent latitudes at $550 \mathrm{~K}$.

QBO shows a strong seasonal dependence. Since the QBO basis function is scaled to $[-1,1]$, the parameter $a_{3}$ added to the climatology $a_{1}$ corresponds to west-phase conditions (i.e. the QBO panels in the figures show the west phase QBOinduced mixing anomaly and with colour scales reversed would show the east phase mixing anomaly). Recall that the regression model allows for a phase shift in the QBO basis function (see Eq. 2). The optimal phase shift, across all latitudes, was found to be -2 months at $450 \mathrm{~K},+2$ months at $550 \mathrm{~K}$ and +6 months at $650 \mathrm{~K}$ as expected from the descending nature of the QBO.

In the lower stratosphere, QBO west phases cause mixing to be enhanced around the equator, with the maximum on the summer side of the equator, from June to October, accompanied by a decrease in mixing in southern subtropics. During the rest of the year, mixing is inhibited between $5^{\circ} \mathrm{S}$ and up to $30^{\circ} \mathrm{N}$. A narrow positive effect is found at $15^{\circ} \mathrm{S}$ whereas mixing is lower in the west phase in the southern subtropics year round. At upper levels, the pattern is more seasonally symmetric: in general QBO parameters at $550 \mathrm{~K}$ and $650 \mathrm{~K}$ show positive values on the summer side of the equator and negative values on the winter side, though in some cases the winter negative values persist into summer in the sub-tropics.

Shuckburgh et al. (2001) investigated the influence of the QBO on isentropic mixing at $624 \mathrm{~K}$ (they argued that the influence is strongest at this level) for 6 years. They found inhibited mixing in east phases throughout the tropics and a narrow band of weak mixing at the equator with regions of enhanced mixing surrounding it during westerly phases. Their calculations were based on equivalent lengths estimated from tracer contours, which were simulated from ECMWF analysed winds. Our results agree, with a broad region of inhibited mixing close to the equator in easterly phases, but with a seasonal shift of the pattern into the summer hemisphere. Both analyses show a strong maximum in mixing on the summer side of the equator in westerly QBO phases. In contrast to Shuckburgh et al. (2001), our analyses show weaker winter-time mixing in the surf zone that does not extend as close to the equator during westerly QBO phases.
Over the Arctic, at all three levels (but less so at $650 \mathrm{~K}$ ), mixing is negatively correlated with the QBO in winter and positively correlated with the QBO in spring. This implies a strengthened Arctic vortex in QBO west phases and a weaker vortex with enhanced mixing in middle to high latitudes in QBO east phases. These results are consistent with Holton and Tan (1980) who showed increased wave activity in winter (November-December) during easterly QBO phases and in late winter/spring (January-March) during westerly QBO phases.

This QBO influence on the Antarctic polar vortex is less obvious though the signal at $650 \mathrm{~K}$ extends far into the Southern Hemisphere.

\subsection{Solar cycle and ENSO influence on mixing}

Both solar cycle and El Niño Southern Oscillation (ENSO) signals are seldom statistically significant in the Lyapunov exponents at all levels (not shown). The only significant signal that lasts for a few months over a broad range of latitudes is a negative correlation of the ENSO with Lyapunov exponents in spring/early summer in northern mid-latitudes at $550 \mathrm{~K}$ and $650 \mathrm{~K}$. This is shown for $650 \mathrm{~K}$ in Fig. 10, where Lyapunov exponents, with the offset, the trend, the QBO and the solar cycle signal removed, and averaged over AprilJune and over $30^{\circ}$ to $60^{\circ} \mathrm{N}$ are plotted against the ENSO index (i.e. the SOI). The correlation between the plotted Lyapunov exponents and SOI is -0.51 , and the correlation is significant at the $99.5 \%$ level. The variability in the averaged Lyapunov exponent residuals is only partly captured by ENSO/SOI (the $R^{2}$ value, see Eq. 5 , of the linear fit is 0.26). However, the ENSO signal in other atmospheric quantities (temperature, ozone) shows strong longitudinal structure (Randel and Cobb, 1994), with the result that the zonal mean analyses presented here may obscure some features. Randel and Cobb (1994) showed a wave-1 pattern in winter high latitudes, which coincides with the strongest signal found in mixing.

\subsection{Zonal averages over equivalent latitudes}

In addition to the zonal averages over geographical latitude, Lyapunov exponents were averaged over latitudes related to potential vorticity (PV) contours i.e. equivalent latitude (Butchart and Remsberg, 1986). The equivalent latitude of a location with potential vorticity $\mathrm{PV}_{\mathrm{loc}}$ is the geographic latitude enclosing the same area as the area over which PV exceeds or equals $\mathrm{PV}_{\text {loc. }}$. The equivalent latitude transformation places the pole at the centre of the vortex and, when calculating zonal means, preserves steep meridional gradients in atmospheric constituents that are correlated with PV. Equivalent latitudes were calculated based on NCEP/NCAR reanalysis PV fields.

The regression model (Eq. 2) was applied to the equivalent latitude averaged Lyapunov exponents, and the variability 

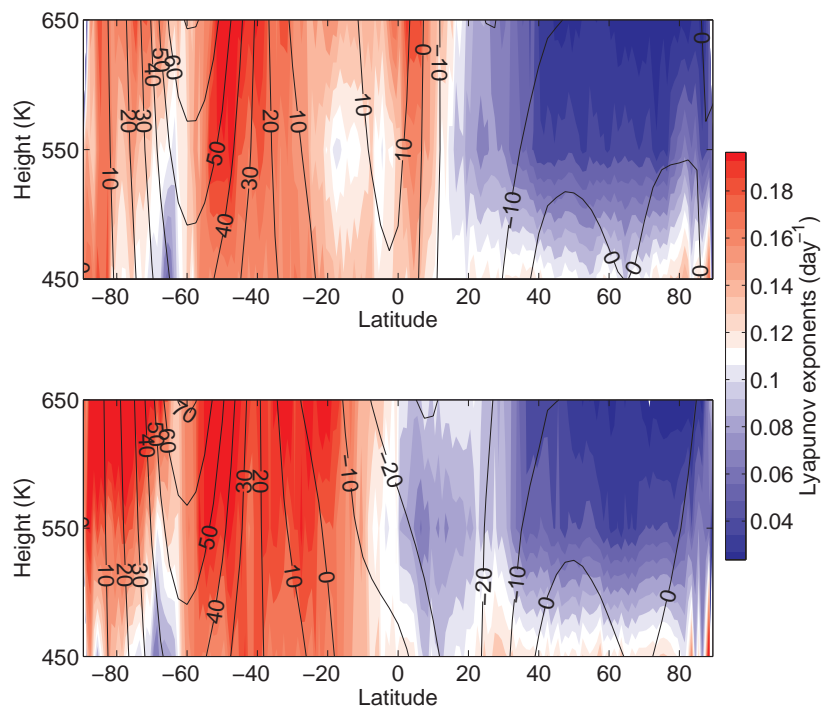

Fig. 12. Latitude-potential temperature cross-sections of Lyapunov exponents (in colour) and of monthly mean zonal mean zonal winds (as contours) for July 1997 (top), representing a month of westerly QBO phase at $50 \mathrm{hPa}$, and July 1984 (bottom), representing a month of easterly QBO phase at $50 \mathrm{hPa}$.

explained by the model was similar to that for true latitude. The climatologies (coefficient $a_{1}$ ) reveal generally steeper gradients of Lyapunov exponents at the vortex edges, both in the Antarctic and the Arctic. The differences are most apparent at $550 \mathrm{~K}$, and the climatology at this level is shown in Fig. 11. The Arctic vortex, which is hardly visible in true latitude coordinates, can be clearly identified as low values in autumn/early winter, extending through the winter with steep gradients at around $60^{\circ} \mathrm{N}$ equivalent latitude. In Antarctica, high values at the vortex edge are concentrated in a narrow band, and with rapid decreases in mixing just equatorward of the vortex edge as the vortex breaks up. The Arctic vortex is apparent also at $450 \mathrm{~K}$ and $650 \mathrm{~K}$ in equivalent latitudes (not shown). The Antarctic vortex and its barrier to mixing is far more clearly visible in equivalent latitude coordinates than its Arctic counterpart. The regression model coefficients $a_{2}$ to $a_{5}$ show only small differences when averaged over equivalent latitude rather than true latitude.

\section{Discussion and conclusions}

The analyses presented above show that Lyapunov exponents appear to be a useful diagnostic of stratospheric mixing: they show regions of strong mixing that coincide with the expected regions of the mid-latitude surf zone, barriers to mixing in the sub-tropics and at the polar vortex edge as shown by steep gradients in PV (see Fig. 2), and the seasonal climatology in mixing revealed by the Lyapunov exponents agrees well with previous studies (e.g. Haynes and Shuck-

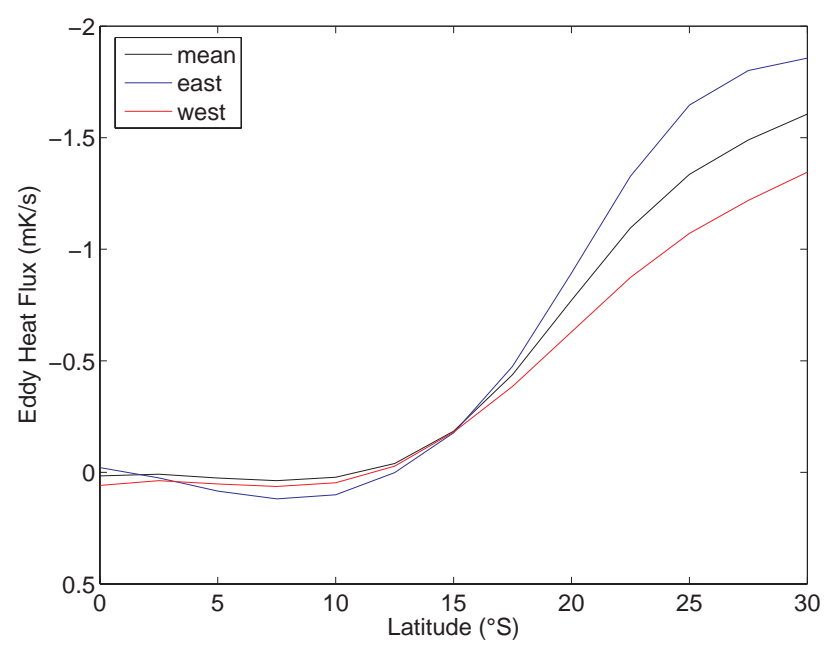

Fig. 13. Monthly mean zonal mean eddy heat flux at $50 \mathrm{hPa}$ for July. The black line shows the average over the years 1979 to 2005, while the red line shows the average over years when the QBO (as defined by the $50 \mathrm{hPa}$ equatorial zonal winds) was westerly in winter $(1980,1985,1990,1997,1999)$ and the blue line when the QBO was easterly $(1979,1984,1989,1994,1996,1998)$.

burgh, 2000; Nakamura, 1996). Averaging over equivalent latitudes shows more clearly the polar barriers, especially in the Northern Hemisphere where the vortex is highly asymmetric and conventional zonal averaging blurs the steep gradients in mixing.

This study, one of the first to present multi-decadal time series of stratospheric mixing, shows long-term secular trends in mixing, interannual variability on time scales coincident with main sources of atmospheric variability e.g. the $\mathrm{QBO}$, as well as unexplained variability ("noise") whose magnitude varies with latitude and altitude. Especially in the tropics and subtropics, mixing is strongly modulated by the QBO, with a strong seasonal dependence. To show the QBO modulation of mixing more clearly, latitude-potential temperature cross-sections in July for two indicative years with opposite QBO phases are shown in Fig. 12 together with monthly mean zonal mean zonal winds.

For July 1997, when the equatorial zonal winds were westerly, the region of enhanced mixing in the summer tropics/subtropics coincides with the zero wind line. This agrees with Shuckburgh et al. (2001) and suggests that in QBO west phases, planetary waves can penetrate from the winter hemisphere into the tropics and break on the summer side of the equator (where winds turn easterly), which is expected to increase mixing there. In July 1984, when zonal winds were easterly throughout the tropics and the zero wind line lies at around $20^{\circ} \mathrm{S}$, planetary waves are expected to break on the winter side of the tropics, which explains the higher mixing in the winter subtropics. The consistency of this behaviour with variability in wave activity is shown in 


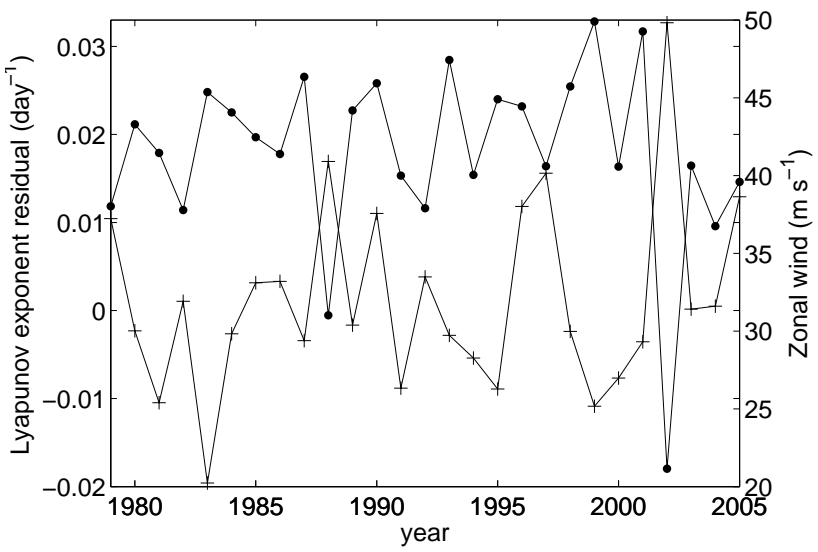

Fig. 14. Residuals of $450 \mathrm{~K}$ Lyapunov exponents after subtracting all terms except the linear trend $\left(a_{2}\right)$, averaged over $60^{\circ}$ to $80^{\circ} \mathrm{S}$ for August of each year (solid line with crosses plotted against the left $\mathrm{y}$-axis) and zonal mean zonal winds at $450 \mathrm{~K}$ and $60^{\circ} \mathrm{S}$ for October (solid line with dots plotted against the right $\mathrm{y}$-axis).

Fig. 13 where $50 \mathrm{hPa}$ July monthly mean zonal mean eddy heat fluxes $\left(\overline{v^{\prime} T^{\prime}}\right)$ for southern low latitudes are shown for different phases of the QBO.

It can be seen that in east phases, $\left|\overline{v^{\prime} T^{\prime}}\right|$ exceeds the mean values whereas in west phases $\left|\overline{v^{\prime} T^{\prime}}\right|$ is weaker than usual south of $15^{\circ} \mathrm{S}$, as expected from the discussion above. However, even though it is assumed that planetary wave breaking is the main cause of mixing, further analyses are required to explore the relationship between planetary wave breaking and mixing diagnostics (in particular Lyapunov exponents), and to determine which other factors contribute to dynamical mixing.

The QBO induced variability in mixing is likely to have an influence on the distribution of long-lived atmospheric trace gases such as ozone. During QBO west phases, a strengthened tropical barrier on the winter side of the equator and enhanced mixing in the summer tropics/subtropics suggests that trace gases, whose source is in the tropics, should show a positive anomaly in the summer subtropics and a negative anomaly in the winter hemisphere. However, because the effect of the QBO on mixing is seasonally dependent, the anomaly in trace gas distributions is sensitive to the phasing of the QBO with respect to the annual cycle. The effects of this phasing on southern mid-latitude total column ozone have been analysed by Bodeker et al. (2007). The suggestion of penetration of planetary waves across the equator Shuckburgh et al. (2001), see discussion above is in agreement with net transport from the winter hemisphere into the summer hemisphere as suggested here and in Bodeker et al. (2007).

A QBO signal in mixing could be identified in northern high-latitudes (strongest at $550 \mathrm{~K}$ ), with decreased mixing in winter and increased mixing in spring during QBO west phases, suggesting a strengthened Arctic vortex in west QBO conditions. As mentioned earlier, this behaviour is consistent with Holton and Tan (1980) and follow-up studies (Dunkerton and Baldwin, 1991; Dameris and Ebel, 1990). The mechanism suggested by Holton and Tan (1980) is a narrowed wave guide for planetary waves in QBO east phases, causing the wave activity to increase in middle to high latitudes. Increased wave activity and the narrowed wave guide increases the Eliassen-Palm (EP) flux (Randel et al., 1987) towards high latitudes, strengthening the wave-driven mean meridional circulation and causing increased subsidence at high latitudes so that the overall effect is to weaken the vortex in east phases. Our results show increased mixing for east phases from the equator to about $30^{\circ} \mathrm{N}$ and for latitudes higher than $55^{\circ} \mathrm{N}$ in mid-winter. From the Holton-Tan mechanism, increased wave activity is expected at mid-latitudes, and since mixing occurs where waves break, the regions of increased mixing over high latitudes supports the concept of enhanced erosion of the polar vortex by planetary wave breaking. Ruzmaikin et al. (2005) calculated differences in EP fluxes carried by wave 1 and wave 2 for west and east phases of the QBO from NCEP/NCAR reanalyses data at $20 \mathrm{hPa}$ and found significantly higher fluxes for wave 1 in early winter (October-December) and lower fluxes in late winter/spring (January-February) in east phases. Significant differences in wave 2 fluxes were found in spring (FebruaryMarch), where fluxes are lower in east phases than in west phases. The change of sign in the difference in wave fluxes (QBO east phases minus west phases) from winter to spring compares well to the change of sign in mixing anomalies found here.

In the Southern Hemisphere, no significant QBO signal in mixing could be detected at high latitudes. However, at $650 \mathrm{~K}$ (and less so at $550 \mathrm{~K}$ ), in winter, the QBO signal extends far into the mid-latitudes $\left(60^{\circ} \mathrm{S}\right)$ and in spring/early summer Lyapunov exponents are enhanced during QBO west phases and reduced during the QBO east phases. If the wave activity in the Southern Hemisphere is modulated as suggested by the Holton-Tan mechanism (increased wave activity in east phases), the modulation of mixing in the midlatitudes rather than at high latitudes suggests that the waves break at lower latitudes than in the Northern Hemisphere. A possible explanation is the comparably stronger polar vortex over Antarctica, causing waves to break at its edge and not, as in the Northern Hemisphere, propagate to high latitudes and break there. Our analyses of eddy heat fluxes at $30 \mathrm{hPa}$ for July (not shown) suggest that values of $\overline{v^{\prime} T^{\prime}}$ averaged over years with $\mathrm{QBO}$ west phases are lower than values of $\overline{v^{\prime} T^{\prime}}$ in QBO easterlies between $40^{\circ} \mathrm{S}$ and $70^{\circ} \mathrm{S}$ (at $60^{\circ} \mathrm{S}$ eddy heat fluxes in QBO westerlies are about one third of eddy heat flux values in QBO easterlies). This finding supports the hypothesis of enhanced erosion of the polar vortex in QBO east phases.

The regression analysis applied to monthly zonal mean Lyapunov exponents revealed trends in mixing over the 
analysis period of 27 years. At $450 \mathrm{~K}$, an increase in mixing in the Southern Hemisphere, mainly in late winter/spring, is the main feature and holds also for shorter analysis periods (e.g. 1979-2000 and 1985-2005), which were calculated to test the sensitivity of the trend to different analysis periods. This trend is consistent with an increase in winter-time wave 1 amplitudes at $60^{\circ} \mathrm{S}$ over the same time period (Bodeker et al., 2007). From 1979 to 2005 zonal winds at $450 \mathrm{~K}$ and $60^{\circ} \mathrm{S}$ in October show a positive trend (see Fig. 14).

Zonal mean zonal winds at $450 \mathrm{~K}$ and $60^{\circ} \mathrm{S}$ in October were correlated with Lyapunov exponents from which all fitted signals, other than the trend, were removed. It was found that the best correlation is obtained with averages over $60^{\circ}-$ $80^{\circ} \mathrm{S}$ in August (correlation coefficient -0.68 , significant at the $99.5 \%$ level). The negative correlation between August Lyapunov exponents and October zonal winds, apparent in Fig. 14, is at odds with the long-term positive trend in both quantities. This suggests that different mechanism drive the year-to-year and long-term interactions between Lyapunov exponents and vortex strength.

The negative correlation on short time scales is likely to be due to increased wave activity enhancing the wave-driven mean meridional circulation which increases subsidence and therefore the temperature at high latitudes and so decreases the vortex strength (as found by Newman et al., 2001, for the Northern Hemisphere). This mechanism therefore represents a response of the vortex strength to mostly unexplained variability in wave driving (the correlation between Lyapunov exponents and zonal winds proves to be even higher when taking only the residual term into account). The long-term trend in the $60^{\circ} \mathrm{S}$ zonal winds is believed to be caused by decreasing polar temperatures due to ozone depletion (Thompson and Solomon, 2002). Therefore, in contrast to the proposed short-term mechanism where mixing influences the vortex strength, on longer time scales the change in zonal winds is likely to influence mixing. Since Lyapunov exponents do not directly measure wave activity, but rather shear and strain rates, higher Lyapunov exponents will result not only from higher wave activity but also from higher shear due to a stronger polar jet. This again highlights the need to explore the physical causes of mixing in more detail.

The trends at $550 \mathrm{~K}$ and $650 \mathrm{~K}$ are consistent with a strengthening of the Antarctic vortex, with decreased mixing in winter at high latitudes and increased mixing in spring. Mixing at the vortex edge is highly correlated with wave activity, quantified here as eddy heat flux (the correlation coefficient for $650 \mathrm{~K}$ Lyapunov exponents with $30 \mathrm{hPa}$ eddy heat flux at $60^{\circ} \mathrm{S}$ is -0.75). At all three analyses levels, a winter/early spring decrease in northern mid-latitude mixing was found. This decrease in mixing is consistent with negative trends in EP fluxes and eddy heat fluxes in the Northern Hemisphere in winter, that have been reported on in several studies (Hadjinicolaou et al., 2005; Randel et al., 2002; Hood and Soukharev, 2005).
Acknowledgements. We would like to thank C.-F. Shih at the National Center for Atmospheric Research and the National Centers for Environmental Prediction for the NCEP/NCAR data. H. Garny was funded through the Deutsche Akademische Austauschdienst (DAAD). This work was conducted within the FRST funded Drivers and Mitigation of Global Change programme (C01X0204).

Edited by: P. Haynes

\section{References}

Allen, D. R. and Nakamura, N.: A seasonal climatology of effective diffusivity in the stratosphere, J. Geophys. Res., 106, 7917-7936, 2001.

Bodeker, G. E., Garny, H., Smale, D., Dameris, M., and Deckert, R.: The 1985 Southern Hemisphere mid-latitude total column ozone anomaly, Atmos. Chem. Phys., 7, 5625-5637, 2007.

Bowman, K.: Large-scale isentropic mixing properties of the Antarctic polar vortex from analyzed winds, J. Geophys. Res., 98, 23 013-23 027, 1993.

Butchart, N. and Remsberg, E. E.: The area of the stratospheric polar vortex as a diagnostic for tracer transport on an isentropic surface, J. Atmos. Sci., 43, 1319-1339, 1986.

Coy, L., Nash, E. R., and Newman, P. A.: Meteorology of the polar vortex: Spring 1997, Geophys. Res. Lett., 24, 2693-2696, 1997.

Dameris, M. and Ebel, A.: The quasi-biennial oscillation and major stratospheric warmings - A three-dimensional model study, Ann. Geophys., 8, 79-85, 1990,

http://www.ann-geophys.net/8/79/1990/.

Dunkerton, T. J. and Baldwin, M. P.: Quasi-biennial modulation of planetary-wave fluxes in the Northern Hemisphere winter, J. Atmos. Sci., 48, 1043-1061, 1991.

Hadjinicolaou, P., Pyle, J. A., and Harris, N. R. P.: The recent turnaround in stratospheric ozone over northern middle latitudes: A dynamical modeling perspective, Geophys. Res. Lett., 32, L12821, doi:10.1029/2005GL022476, 2005.

Haynes, P. and Shuckburgh, E.: Effective diffusivity as a diagnostic of atmospheric transport 1. Stratosphere, J. Geophys. Res., 105, $22777-22$ 794, 2000.

Holton, J. R. and Tan, H.-C.: The influence of the equatorial quasibiennial oscillation on the global circulation at $50 \mathrm{mb}$, J. Atmos. Sci., 37, 2200-2208, 1980.

Hood, L., Rossi, S., and Beulen, M.: Trends in lower stratospheric zonal winds, Rossby wave breaking behaviour, and column ozone at northern midlatitudes, J. Geophys. Res., 104, 24 32124339, 1999.

Hood, L. L. and Soukharev, B. E.: Interannual Variations of Total Ozone at Northern Midlatitudes Correlated with Stratospheric EP Flux and Potential Vorticity, J. Atmos. Sci., 62, 3724-3740, 2005.

Hoskins, B. J., McIntyre, M. E., and Robertson, A. W.: On the use and significance of isentropic potential vorticity maps, Q. J. R. Meteorol. Soc., 111, 877-946, 1985.

Joseph, B. and Legras, B.: On the relation between kinematic boundaries, stirring, and barriers for the Antarctic polar vortex, J. Atmos. Sci., 59, 1198-1212, 2002.

Kalnay, E., Kanamitsu, M., Kistler, R., Collins, W., Deaven, D., Gandin, L., Iredell, M., Saha, S., White, G., Woollen, J., Zhu, Y., Leetmaa, A., Reynolds, B., Chelliah, M., Ebisuzaki, W., Higgins, 
W., Janowiak, J., Mo, K. C., Ropelewski, C., Wang, J., Jenne, R., and Joseph, D.: The NCEP/NCAR 40-Year Reanalysis Project, Bull. Am. Soc., 77(3), 437-472, 1996.

Koh, T. Y. and Plumb, R. A.: Lobe dynamics applied to barotropic Rossby wave breaking, Phys. Fluids, 12, 1518-1528, 2000.

McIntyre, M. and Palmer, T.: The "surf zone" in the stratosphere, J. Atmos. Terr. Phys., 46, 825-849, 1984.

McKenna, D., Konopka, P., Grooß, J.-U., Günther, G., and Müller, R.: A new Chemical Lagrangian Model of the Stratosphere (CLaMS) 1. Formulation of advection and mixing, J. Geophys. Res., 107, doi:10.1029/2000JD000114, 2002.

Nakamura, N.: Two-dimensional mixing, edge formation, and permeability diagnosed in an area coordinate, J. Atmos. Sci., 53, 1524-1537, 1996.

Nakamura, N. and Ma, J.: Modified Lagrangian-mean diagnostics of the stratospheric polar vortices 2 . Nitrous oxide and seasonal barrier migration in the cryogenic limb array etalon spectrometer and SKYHI general circulation model, J. Geophys. Res., 102, 25 721-25 735, 1997.

Newman, P. A., Nash, E. R., and Rosenfield, J. E.: What controls the temperature of the Arctic stratosphere during the spring?, J. Geophys. Res., 106, 19 999-20 010, 2001.

Newman, P. A. and Nash, E. R.: The Unusual Southern Hemisphere Stratosphere Winter of 2002, J. Atmos. Sci., 62, 614-628, 2005.

Pierrehumbert, R. T. and Yang, H.: Global Chaotic Mixing on Isentropic Surfaces, J. Atmos. Sci., 50, 2462-2480, 1993.

Plumb, R. A. and Ko, M. K. W.: Interrelationships between mixing ratios of long-lived stratospheric constituents, J. Geophys. Res., 97, 10 145-10 156, 1992.

Press, W. H., Flannery, B. P., Teukolsky, S. A., and Vetterling, W. T.: Numerical recipes in Pascal. The art of scientific computing, Cambridge University Press, 1989.

Randel, W. J., Stevens, D. E., and Stanford, J. L.: A study of planetary waves in the southern winter troposphere and stratosphere. Part II: Life cycles, J. Atmos. Sci., 44, 936-949, 1987.

Randel, W. and Cobb, J.: Coherent variations of monthly mean total ozone and lower stratospheric temperature, J. Geophys. Res., 99, 5433-5447, 1994.

Randel, W. J., Wu, F., and Stolarski, R.: Changes in column ozone correlated with the stratospheric EP flux, J. Meteorol. Soc. Japan, 80, 849-862, 2002.
Rosenstein, M. T., Collins, J. J., and de Luca, C. J.: A practical method for calculating largest Lyapunov exponents from small data sets, Physica D, 65, 117-134, 1993.

Ruzmaikin, A., Feynman, J., Jiang, X., and Yung, Y. L.: Extratropical signature of the quasi-biennial oscillation, J. Geophys. Res., 110, D11111, doi:10.1029/2004JD005382, 2005.

Shuckburgh, E. and Haynes, P.: Diagnosing transport and mixing using a tracer-based coordinate system, Phys. Fluids, 15, 33423357, 2003.

Shuckburgh, E., Norton, W., Iwi, A., and Haynes, P.: Influence of the quasi-biennial oscillation on isentropic transport and mixing in the tropics and subtropics, J. Geophys. Res., 106, 1432714337, 2001.

Tan, D. G. H., Haynes, P. H., MacKenzie, A. R., and Pyle, J. A.: Effects of fluid-dynamical stirring and mixing on the deactivation of stratospheric chlorine, J. Geophys. Res., 103, 1585-1605, 1998.

Thompson, D. W. J. and Solomon, S.: Interpretation of recent Southern Hemisphere climate change, Science, 296, 895-899, 2002.

Tiao, G., Reinsel, G., Xu, D., Pedrick, J., Zhu, X., Miller, A., DeLuisi, J., Mateer, C., and Wuebbles, D.: Effects of autocorrelation and temporal sampling schemes on estimates of trend and spatial correlation, J. Geophys. Res., 95, 20 507-20 517, 1990.

Trepte, C. R. and Hitchman, M. H.: Tropical stratospheric circulation deduced from satellite aerosol data, Nature, 355, 626-628, 1992.

van Loon, H. and Labitzke, K.: The Influence of the 11-year Solar Cycle on the Stratosphere Below $30 \mathrm{~km}$ : a Review, Space Sci. Rev., 94, 259-278, 2000.

von Storch, H. and Zwiers, F. W.: Statistical Analysis in Climate Research, pp. 494, ISBN 0521012309, Cambridge, UK, Cambridge University Press, 2002.

Waugh, D. W.: Subtropical stratospheric mixing linked to disturbances in the polar vortices, Nature, 365, 535-537, 1993.

Waugh, D., Plumb, R., Atkinson, R. J., Schoeberl, M. R., Lait, L. R., Newman, P. A., Loewenstein, M., Toohet, D., Avallone, L., Webster, C., and May, R.: Transport out of the lower stratospheric vortex by Rossby wave breaking, J. Geophys. Res., 99, 1071-1088, 1994.

Wolf, A., Swift, J. B., Swinney, H. L., and Vastano, J. A.: Determining Lyapunov exponents from a time series, Physica D, 16, 285-317, 1985. 